\title{
3 Schritte für eine bessere Mundhygiene
}

Basis für den langfristigen Erhalt der Zahngesundheit ist ein gutes Biofilmmanagement. Eine aktuelle, länderübergreifende Meinungsumfrage mit 4100 Teilnehmern zeigt: Deutlich mehr als die Hälfte der Patienten sind bereit, ihre Mundhygiene zu verbessern, auch wenn dazu ein zusätzlicher Schritt in der täglichen Mundpflege nötig ist. Auf dieser Erkenntnis beruht die aktuelle ListerineKampagne „Sei bereit für alles“. Sie zeigt Menschen, die mehr wollen im Leben auch in Sachen Mundgesundheit. Eine klare zahnmedizinische Beratung kann den entscheidenden Impuls für eine bessere Mundhygiene geben: Die tägliche 3-fach-Prophylaxe bestehend aus Zähneputzen, Interdentalraumreinigung und der Anwendung einer antibakteriellen
Mundspülung (z.B. Listerine) verbessert die Mundhygiene der Patienten. ListerineMundspülungen enthalten bis zu 4 ätherische Öle (Eukalyptol, Thymol, Menthol und Methylsalicylat). Diese sind dazu in Lage, den dentalen Biofilm zu durchdringen, und können somit dabei helfen, pathogene Keime effektiv zu bekämpfen. Mehr Informationen zur „Sei bereit“Kampagne gibt es auf www.listerine.de.

Nach einer Pressemitteilung der Johnson \& Johnson GmbH, Neuss

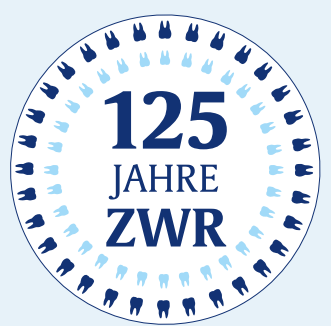

